\title{
Whole-Exome Sequencing Identifies Three Candidate Homozygous Variants in a Consanguineous Iranian Family with Autism Spectrum Disorder and Skeletal Problems
}

\author{
Saeed Farajzadeh Valilou ${ }^{a}$ Afagh Alavi $^{\mathrm{a}}$ Mahdiyeh Pashaei ${ }^{\mathrm{a}}$ \\ Saghar Ghasemi Firouzabadi ${ }^{a}$ Yousef Shafeghatia, b Ahoura Nozari $^{a}$

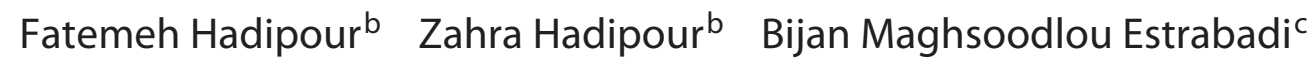 \\ Seyed Gholamreza Noorazar ${ }^{d}$ Susan Banihashemi ${ }^{a} \quad J_{a v a d}$ Karimian ${ }^{a}$ \\ Mahshid Fattahi ${ }^{a}$ Farkhondeh Behjati ${ }^{a}$

\begin{abstract}
a Genetics Research Center, University of Social Welfare and Rehabilitation Sciences, Tehran, Iran; ${ }^{b}$ Department of Medical Genetics and Sarem Cell Research Center (SCRC), Sarem Womens' Hospital, Tehran, Iran; 'Department of Psychiatry, University of Social Welfare and Rehabilitation Sciences, Tehran, Iran; ${ }^{d}$ Research Center of Psychiatry and

Behavioral Sciences, Tabriz University of Medical Sciences, Tabriz, Iran
\end{abstract}

\section{Keywords}

Autism spectrum disorder - FBN1 - PLOD2 - TF . Whole-

exome sequencing

\begin{abstract}
Autism spectrum disorder (ASD) is characterized by 3 core symptoms with impaired social communication, repetitive behavior, and/or restricted interests in early childhood. As a complex neurodevelopmental disorder (NDD), the phenotype and severity of autism are extremely heterogeneous. Genetic factors have a key role in the etiology of autism. In this study, we investigated an Azeri Turkish family with 2 ASD-affected individuals to identify probable ASD-causing variants. First, the affected individuals were karyotyped in order to exclude chromosomal abnormalities. Then, wholeexome sequencing was carried out in one affected sibling followed by cosegregation analysis for the candidate variants in the family. In addition, SNP genotyping was carried
\end{abstract}

out in the patients to identify possible homozygosity regions. Both proband and sibling had a normal karyotype. We detected 3 possible causative variants in this family: c.5443G>A; p.Gly1815Ser, c.1027C>T; p.Arg343Trp, and c.382A > G; p.Lys128Glu, which are in the FBN1, TF, and PLOD2 genes, respectively. All of the variants cosegregated in the family, and SNP genotyping revealed that these 3 variants are located in the homozygosity regions. This family serves as an example of a multimodal polygenic risk for a complex developmental disorder. Of these 3 genes, confluence of the variants in FBN1 and PLOD2 may contribute to the autistic features of the patient in addition to skeletal problems. Our study highlights the genetic complexity and heterogeneity of NDDs such as autism. In other words, in some patients with ASD, multiple rare variants in different loci rather than a monogenic state may contribute to the development of phenotypes.

\section{KARGER}

(C) 2020 S. Karger AG, Basel

karger@karger.com

www.karger.com/msy
Prof. Farkhondeh Behjati

Genetics Research Center, University of Social Welfare and Rehabilitation Sciences Kodakyar Ave., Daneshjo Blvd., Evin

Teheran 1985713871 (Iran)

f_behjati@uswr.ac.ir 
Autism spectrum disorder (ASD) is a neurodevelopmental disorder which is characterized by features including communication deficits, abnormal social interests, and restricted and repetitive behaviors. The fifth edition of the Diagnostic and Statistical Manual of Mental Disorders (DSM-5) has considered autism subtypes listed in DSM-4 including Asperger disorder, pervasive developmental disorder not otherwise specified, and childhood disintegrative disorder under a single category named ASD [American Psychiatric Association, 2013]. The prevalence of ASD in the most recent surveys in 2018 is estimated to be 1 in 59 children (about 1.5\%) in developed countries, which presents an increase compared to the prevalence of ASD in 2014 (1 in 68) [Lyall et al., 2017; Baio et al., 2018].

It has been known that in causing ASD not only environmental factors (such as prenatal exposure to air pollution and short interpregnancy interval, certain prenatal nutrients, metabolic conditions, and exposure to endocrine-disrupting chemicals) are involved, but also genetic factors are of paramount importance. To support the major role of genetic factors in the etiology of ASD, it has been shown that concordance rates of ASD among siblings, monozygotic twins, and dizygotic twins are $3-26,50-90$, and $0-30 \%$, respectively [Berg and Geschwind, 2012; Lyall et al., 2017]. Less than $20 \%$ of individuals with ASD, however, have an identifiable genetic etiology corresponding to known single gene disorders or chromosomal rearrangements [De Rubeis and Buxbaum, 2015]. Autistic behaviors may also occur as a part of monogenic syndromes (e.g., Fragile X syndrome, Rett syndrome) or syndromes due to chromosomal abnormalities [Devlin and Scherer, 2012; De Rubeis and Buxbaum, 2015]. Simons Foundation Autism Research Initiative (SFARI) Human Gene Module lists 1,007 genes implicated in autism, of which 68 genes contribute to the syndromic form of ASD (https://gene.sfari.org/database/ human-gene/).

Next-generation sequencing (NGS) technology in comparison to other techniques has less bias for common variants; therefore, this technology detects genetic variants at genome-wide level effectively and is applicable in personalized medicine [Tucker et al., 2009]. Wholeexome sequencing (WES) has been widely used to identify genetic causes or related variations in autism. However, identification of disease-causing genes - only using WES - in heterogenic disorders is challenging. Applying homozygosity mapping in highly heterogeneous diseases such as autism may facilitate finding the causative genes in the patients, especially in consanguineous families.

Three Variants in a Family with ASD and Skeletal Problems
In the present study, WES technique was used to identify rare variants in an Iranian Azeri (Turkish) consanguineous family with 2 affected ASD individuals. In this regard, we analyzed WES data in order to exclude false positives and benign polymorphisms from the NGS results in highly heterogeneous diseases. We also carried out homozygosity mapping in the patients to identify homozygous regions in the entire genome.

\section{Case Presentations}

We present an Iranian Azeri Turk family with 2 ASD individuals (Fig. 1). The parents are related. The 17-year-old proband $(\mathrm{IV}: 1)$ is the first child of unaffected parents. His brother (IV:2) is also affected with ASD. At birth, the proband had jaundice for 3 days. His weight was $3.5 \mathrm{~kg}$, height was $50 \mathrm{~cm}$, and head circumference was $36 \mathrm{~cm}$ at birth. The proband suffered from speech delay. He presented with unbalanced movements and repetitive behaviors. He is totally dependent on his parents requiring support in all activities of daily life. Gross motor development was not normal (sitting at 1 year and walking at 3 years of age), and he has difficulty changing positions. He started to speak (words) at the age of 4 years. DSM-5 criteria were used in diagnosis of the cases. Also, autism behavior checklist was performed, and the score was calculated as 126 for the proband with a minimum cutoff of 68. Clinical examination revealed specific dysmorphic features including micrognathia and macrocephaly. He has arachnodactyly. This boy also has a flat, round face; posteriorly rotated large ears; synophrys, and a short philtrum. In addition, he has unilateral simian crease. He also suffers from asthma.

The 9-year-old affected brother shares most of the signs with the proband. He also has speech delay, unbalanced movements, micrognathia, posteriorly rotated ears, and a short philtrum. ASD assessment using the autism behavior checklist revealed a score of 129. His weight was $3.25 \mathrm{~kg}$, height was $50 \mathrm{~cm}$, and head circumference was $36 \mathrm{~cm}$ at birth. Gross motor development was abnormal, all developmental milestones (sitting, standing and walking) were delayed; a toe-walking pattern was observed. He has difficulty changing positions as well. He started to speak well, but later he experienced speech regression. The same as the proband, he has difficulty eating, drawing, dressing, playing, and writing, and is dependent on his parents. He also has a deep nasal bridge and skin rash. He experienced frequent fractures of the arms and legs. Laboratory tests revealed a calcium and vitamin D deficiency, which are being treated. Frequent seizures had also been observed in the affected brother. None of the features are present in the parents (III:3 and III:4). Unfortunately, the family did not give consent to take photos.

\section{Materials and Methods}

Sampling, DNA Extraction, and Karyotyping

Peripheral whole blood was obtained from the affected and unaffected family members. DNA was isolated from leukocytes by standard salting-out method. In addition, heparinized blood was 


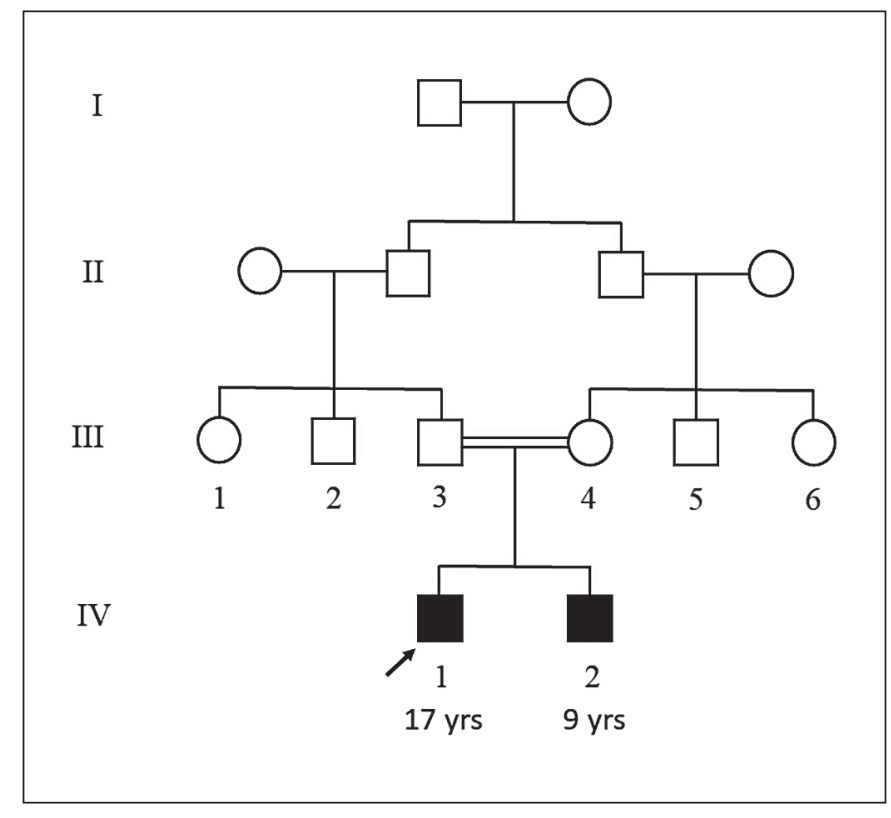

Fig. 1. Pedigree of the family with consanguineous marriage with an autosomal recessive pattern of inheritance. The proband is indicated by an arrow.

obtained from the affected individuals for cytogenetic analysis. Karyotyping was performed with a resolution of 490-540 bands in order to exclude the patients with chromosomal abnormalities from the study.

\section{Whole-Exome Sequencing}

WES was performed on patient IV:1. DNA samples were prepared, and the Agilent SureSelect V6-Post kit (Agilent Technologies, Inc., Santa Clara, CA, USA) was used to prepare exome library of the proband. Subsequently, the sample was sequenced via Illumina HiSeq4000 instrument with the average read depth of $100 \times$ for the targeted platform. Sequence alignment was carried out with the human reference genome GRCh37/hg19 build using the Burrows-Wheeler Alignment Tool (BWA-0.7.12).

\section{Bioinformatics Analysis of Whole-Exome Data}

Duplicates of PCR were excluded using Picard (picardtools-1.130). Genome Analysis Toolkit (GATKv3.4.0) was used for variant calling and local realignment, and quality recalibration was performed. Finally, annotation of variants was performed using wANNOVAR software (http://wannovar.wglab.org/). After variant detection, variations located in down/upstream, intronic, intergenic, $5^{\prime}$ and $3^{\prime}$ UTRs, and synonymous variations were excluded. In this study, variants with minor allele frequency greater than 0.01 were considered as common variants and excluded according to the following databases including the 1000 Genomes project (www.1000genomes.org), Exome Aggregation Consortium (ExAc) (http://exac.broadinstitute.org/), Genome Aggregation Database (gnomAD) (http://gnomad.broadinstitute.org), and Exome Sequencing Project (ESP) (http://evs.gs.washington.edu/EVS/). Furthermore, the frequency of variants is also assessed using an in-

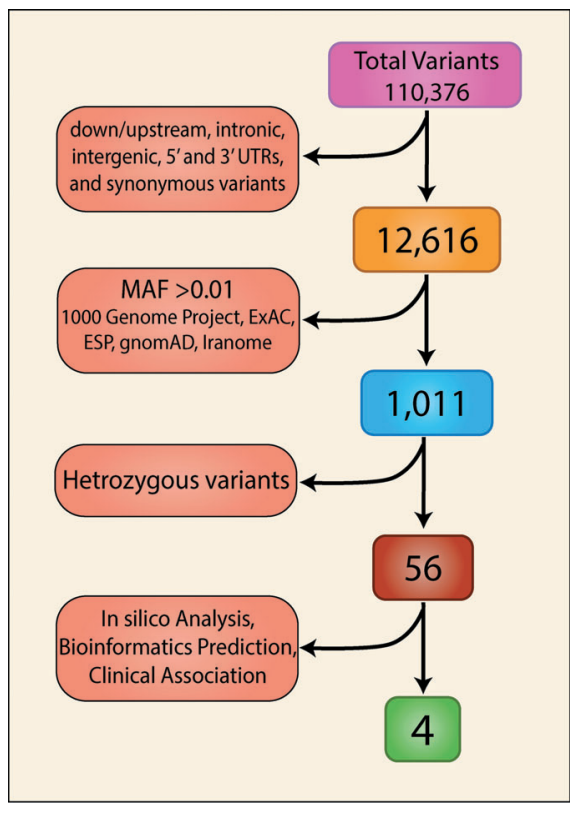

Fig. 2. Flow chart identifying candidate variants in the affected patients.

house Iranome database (http://iranome.com/) including 800 healthy Iranian individuals, and minor allele frequency $>0.01$ in this database was removed. Afterwards, remained rare variants were classified according to in silico protein function prediction scores in SIFT (http://sift.bii.astar.edu.sg/), PolyPhen-2 (http://genetics.bwh. harvard.edu/pph2/), MutationTaster (www.mutationtaster.org), MutationAssessor (http://mutationassessor.org/r3/), FATHMM (http://fathmm.biocompute.org.uk/), PROVEAN (http://provean. jcvi.org/index.php), CADD (http://cadd.gs.washington.edu/), and GERP++ (UCSC Genome Browser). In the next step, gene-based arrangements incorporating evolutionary conservation scores of the variations using SiPhy_29way_logOdds and PhyloP scores were obtained. Then, variations were selected based on mode of the inheritance of the disease in the pedigree. Finally, we looked for variants that may be involved in pathogenicity of autism and/or neurodevelopmental disorders using expression pattern, protein function and related pathways as well as special databases for autism, such as SFARI (https://www.sfari.org/) and AutismKB (http://autismkb. cbi.pku.edu.cn).

Sanger Sequencing Validation and Segregation Analysis

Validation and segregation testing of the candidate variants were performed using PCR on the proband and other family members. Primers were designed and PCR products proceeded Sanger sequencing. Ultimately, the data were analyzed for mutation confirmation and cosegregation.

\section{SNP Genotyping}

SNP genotyping was carried out using Illumina CytoSNP$12 \mathrm{v} 2.1330 \mathrm{~K}$ arrays following the Infinium ${ }^{\circledR} \mathrm{HD}$ Assay Ultra manual protocol. The Illumina GenomeStudio Integrated Informatics 
Table 1. List of candidate variants for cosegregation analysis

\begin{tabular}{|c|c|c|c|c|c|c|c|c|c|c|c|c|}
\hline Gene & Coordinates & Variant & dbSNP ID & $\begin{array}{l}\text { MAF in } \\
\text { gnomAD }\end{array}$ & $\begin{array}{l}\text { SIFT_ }_{-} \\
\text {pred }\end{array}$ & $\begin{array}{l}\text { PolyPhen-2 } \\
\text { HDIV_pred }\end{array}$ & $\begin{array}{l}\text { MutationTaster_ } \\
\text { pred }\end{array}$ & $\begin{array}{l}\text { MutationAssessor_ } \\
\text { pred }\end{array}$ & $\begin{array}{l}\text { FATHMM_ } \\
\text { pred }\end{array}$ & $\begin{array}{l}\text { CADD_ } \\
\text { pred }\end{array}$ & GERP++ & SiPhy \\
\hline ABCB6 & $\begin{array}{l}\text { Chr 2: } \\
220080864\end{array}$ & $\begin{array}{l}\text { NM_005689.2 } \\
\text { c.1009G }>\text { A; } \\
\text { p.Val337Met }\end{array}$ & Novel & 0.00003233 & $\mathrm{D}$ & $\mathrm{D}$ & $\mathrm{D}$ & $\mathrm{H}$ & $\mathrm{D}$ & 32 & 5.07 & 19.014 \\
\hline FBN1 & $\begin{array}{l}\text { Chr 15: } \\
48744861\end{array}$ & $\begin{array}{l}\text { NM_000138 } \\
\text { c.5443G>A; } \\
\text { p.Gly1815Ser }\end{array}$ & rs745680336 & 0.00001626 & $\mathrm{~T}$ & D & $\mathrm{D}$ & $\mathrm{N}$ & $\mathrm{D}$ & 28.3 & 5.49 & 19.339 \\
\hline PLOD2 & $\begin{array}{l}\text { Chr 3: } \\
145828192\end{array}$ & $\begin{array}{l}\text { NM_182943 } \\
\text { c.382A>G; } \\
\text { p.Lys128Glu }\end{array}$ & rs200569129 & 0.0006541 & $\mathrm{D}$ & D & $\mathrm{D}$ & M & $\mathrm{T}$ & 27.5 & 5.16 & 15 \\
\hline$T F$ & $\begin{array}{l}\text { Chr 3: } \\
133476769\end{array}$ & $\begin{array}{l}\text { NM_001063 } \\
\text { c.1027C>T; } \\
\text { p.Arg343Trp }\end{array}$ & rs150854910 & 0.0008660 & $\mathrm{D}$ & D & $\mathrm{N}$ & M & $\mathrm{T}$ & 31 & 3.12 & 13.469 \\
\hline
\end{tabular}

D, deleterious; $H$, high; $M$, medium; MAF, minor allele frequency; $N$, neutral; T, tolerated.

Table 2. Primer sequences for amplification and sequencing of ABCB6, FBN1, PLOD2, and TF exonic regions containing the variants

\begin{tabular}{llll}
\hline Gene & Forward primer $\left(5^{\prime} \rightarrow 3^{\prime}\right)$ & Reverse primer $\left(5^{\prime} \rightarrow 3^{\prime}\right)$ & Amplicon size, bp \\
\hline ABCB6 & GTATGAGAGACTCCGCCCTC & ACATGCCCTTTCCACTGG & 393 \\
FBN1 & GTGAGAGGCTTTGTTGACTGG & GCAGCAGACAGGCAAATACG & 462 \\
TFOD2 & GTTGGCACAGGTATTAATCTTGC & TCTTCCAGTATCTAACTCACCTCC & 309 \\
\hline
\end{tabular}

Platform was used to extract, visualize, and analyze the genotyping data. The data were then analyzed, looking for notable regions of homozygosity (typically $>1 \mathrm{Mb}$ ) and to compare genotyping data across samples and patients.

\section{Results}

\section{Genomic, in silico, and Cosegregation Analyses}

Using bioinformatics filtering strategies and in silico analysis, 4 candidate variants were selected to be confirmed by Sanger sequencing in the proband (Fig. 2; Table 1). In this regard, we designed primers for each of the 4 variants in order to perform PCR and then sequence the PCR products (Table 2). Further cosegregation analysis followed using other samples including the affected brother (IV:2) and the parents (III:3 and III:4). Of 4 candidate variants in $A B C B 6, F B N 1, P L O D 2$, and $T F$ genes, 3 homozygous variants including FBN1 (NM_000138, c.5443G $>$ A; p.Gly1815Ser), PLOD2 (NM_182943, c.382A>G; p.Lys128Glu) and TF (NM_001063, c.1027C>T; p.Arg343Trp) were cosegregated in this family. The proband and the affected brother were homozygous for both of the variants, and their parents presented heterozygous condition (Fig. 3).

\section{SNP Genotyping Results}

Genome-wide SNP genotyping in this family identified 4 regions in chromosomes 3,15, and 16 (2 regions) that were homozygous in the affected individuals (Fig. 4; Table 3). The variants corresponding to these regions were extracted from the exome data. Nine variants with our filtering criteria remained (Table 4). Six variants were either nonpathogenic variants (based on in silico analysis) or we did not find any association between their function and the phenotypic features of our patients. Finally, 3 variants in the PLOD2, TF, and FBN1 genes were selected as candidate variants in the ASD family.

\section{Discussion}

In this study, we performed WES on an Iranian Azeri Turkish family with 2 affected siblings who not only present with autism, but also manifest bone and skeletal abnormalities. Taking the SNP genotyping results into account, we identified 3 candidate variants FBN1, PLOD2, and TF. In the following, we will pinpoint and 
Fig. 3. DNA sequence chromatograms showing the variants in the FBN1, PLOD2, and TF genes. IV:1 and IV:2 show the affected individuals; III-3 and III-4 show the unaffected parents.

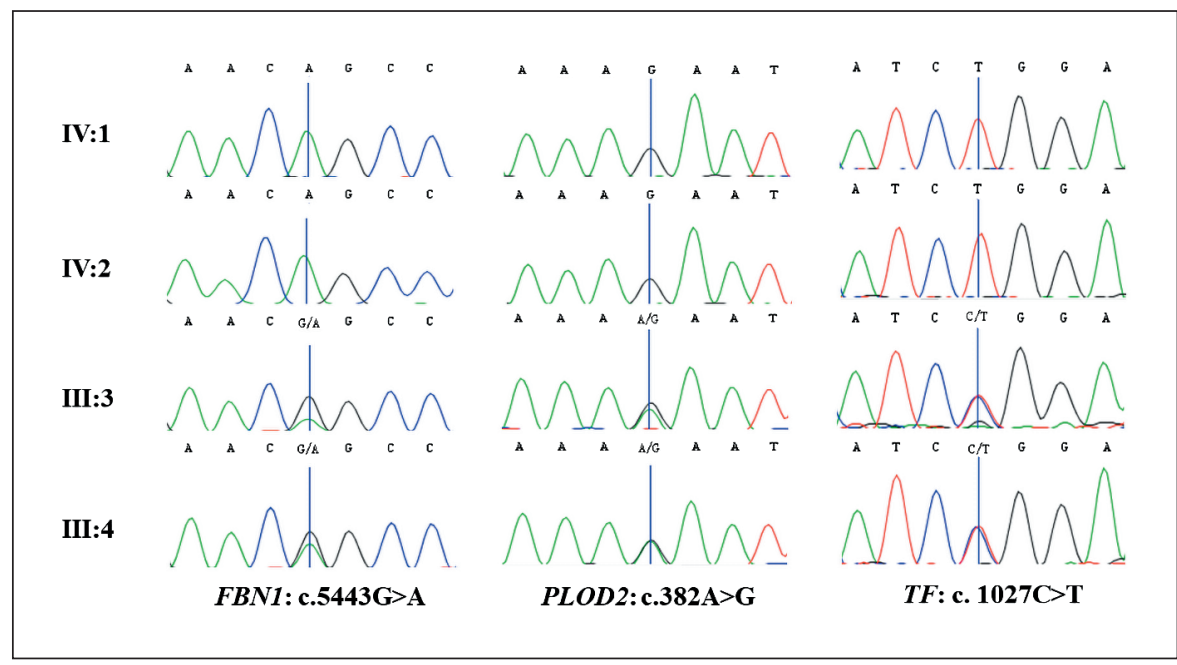

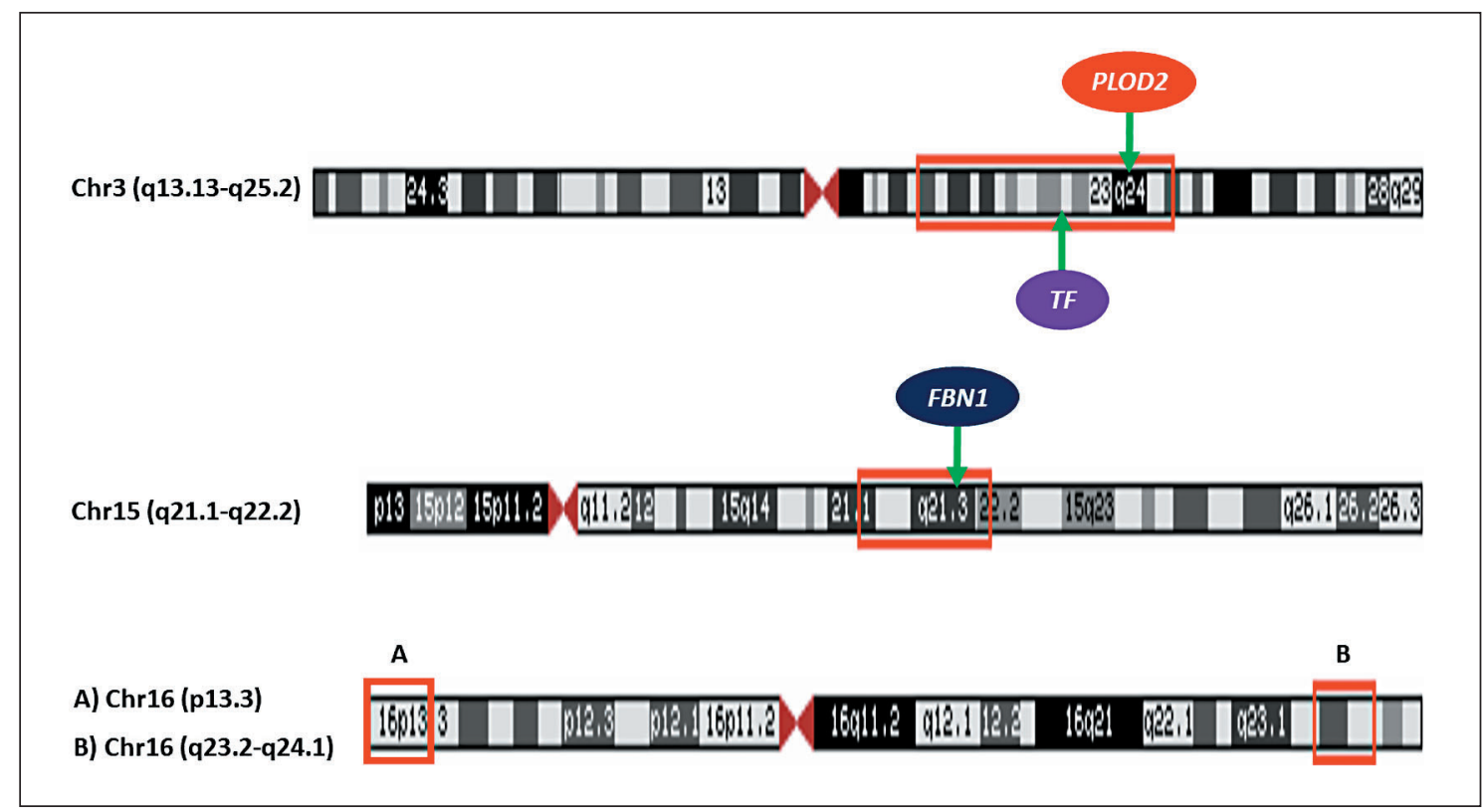

Fig. 4. Position of homozygous regions in chromosomes 3,15 , and 16 . Red boxes indicate the homozygous regions in chromosome 3 including TF and PLOD2, in chromosome 15 including FBN1, and in chromosome 16 without any corresponding genes from exome data.

discuss each of the genes and elaborate their possible role in the manifestation of the clinical features of the patients.

\section{FBN1}

The major constitutive element of extracellular microfibrils is fibrillin (encoded by FBN1) which is distributed widely in both elastic and nonelastic connective tissue throughout the body (OMIM 134797). Microfibrils containing fibrillin-1 provide long-term force-bearing structural support. The periphery of the elastic fiber is formed by microfibrils in tissues such as skin, blood vessels, and lung. Fibrillin-1 also has a critical role in homeostasis of tissue through specific interactions with growth factors, such as the growth and differentiation factors and latent transforming growth factor-beta-binding proteins, bone
66

Mol Syndromol 2020;11:62-72 DOI: $10.1159 / 000506530$
Farajzadeh Valilou et al. 
Table 3. Homozygosity regions identified in both affected individuals (IV:1 and IV:2)

\begin{tabular}{rlllrr}
\hline Chromosome & Start & End & SNP, start-end & Size, Mb & Genes in the region, $n$ \\
\hline 3 & 108009988 & 153580135 & rs709441-rs902432 & 45.57 & 776 \\
15 & 47664559 & 60312237 & rs4774501-rs10152968 & 12.65 & 202 \\
16 & 81210897 & 86348726 & rs12596232-rs12448818 & 5.14 & 95 \\
16 & 1 & 4970538 & rs216596-rs9940799 & 4.97 & 314 \\
\hline
\end{tabular}

Table 4. Variants corresponding to homozygosity region in chromosomes 3, 15, and 16

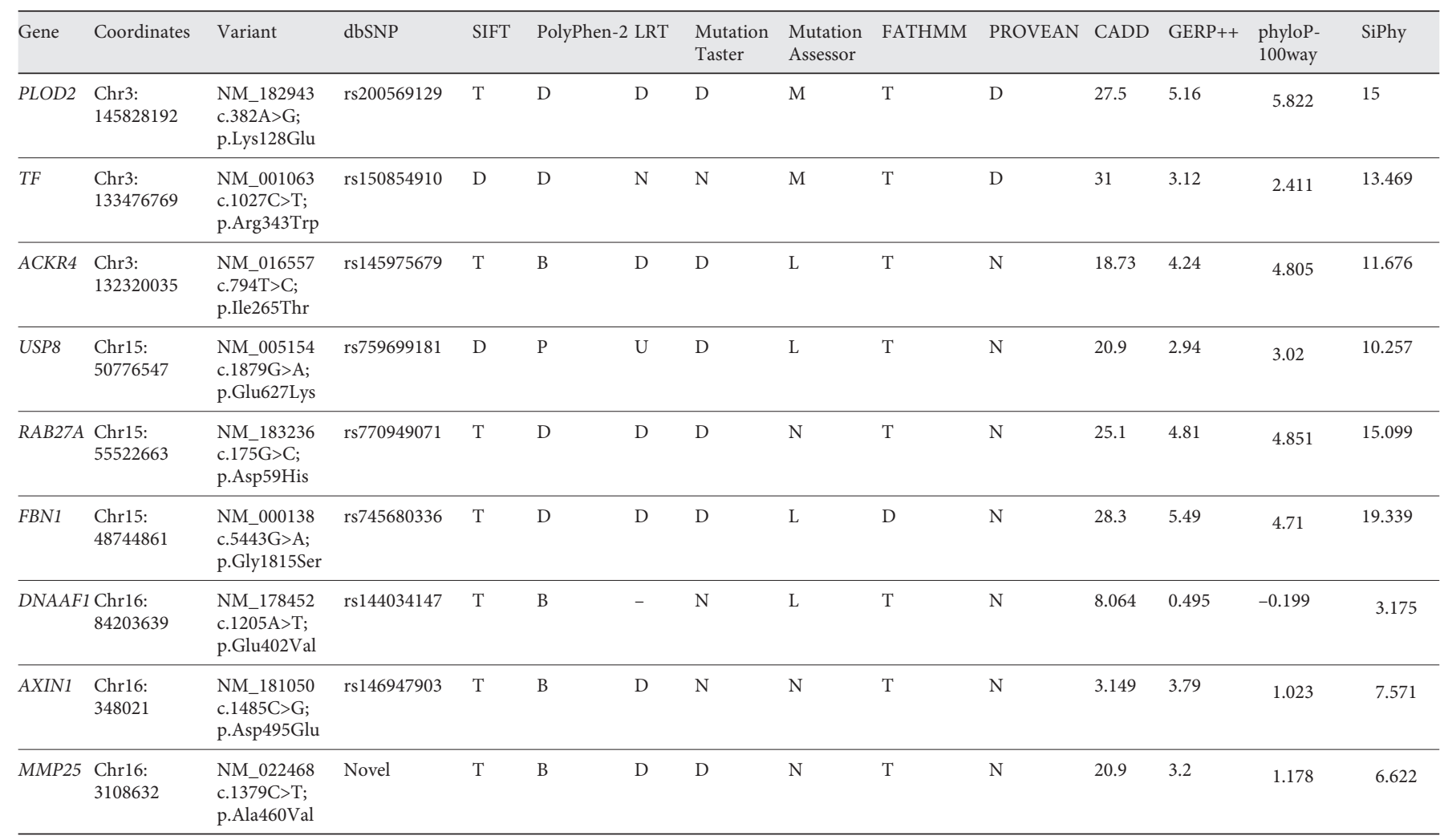

B, benign; D, deleterious; L, low; M, medium; N, neutral; P, possibly damaging; T, tolerated; U, unknown.

morphogenetic proteins, cell-surface integrins, and other protein and proteoglycan components of the extracellular matrix [Jensen and Handford, 2016]. Fibrillin-1 regulates osteoblast maturation by controlling TGF-beta bioavailability and calibrating TGF-beta and bone morphogenetic protein levels [Bax et al., 2003; Jovanovic et al., 2007].

FBN1 gene variants lead to different types of diseases including Marfan syndrome (MFS; OMIM 616914), familial Ectopia lentis (OMIM 129600), Acromicric dysplasia (OMIM 102370), and Weill-Marchesani syndrome 2 (OMIM 608328). Various studies have confirmed that mutations in the FBN1 gene are the major cause of MFS. In this study, we identified a missense variant c.5443G $>A$ (p.Gly1815Ser, rs745680336), which has been reported previously as a likely pathogenic variant in ClinVar (https://www.ncbi.nlm.nih.gov/clinvar/variation/200064/). As previously mentioned, the patients in this study present arachnodactyly and facial features such as micrognathia; these signs are also present in patients with MFS [Dean, 2007; Faivre et al., 2007] (OMIM 154700). Cardiovascular problems, which is a key feature of MFS, was not observed in our patients, suggesting that variants 
in FBN1 may present a variable and broad phenotypic spectrum of clinical manifestations [Reyes-Hernández et al., 2016], and apparently normal parents do not show clinical features due to incomplete penetrance.

Two de novo missense variants, c.4057G $>A$ (p. Gly1353Arg) and c.3971C>G (p.Asn1324Ser), in FBN1 were identified in ASD probands from the Simons Simplex Collection [Iossifov et al., 2012, 2014]. Another de novo missense variant, c.6917G >A (p.Arg2306His), in this gene was identified in a study by De Rubeis et al. [2014]. However, in a study by Krumm et al. [2015], it was reported that no de novo single nucleotide variations (SNVs) in this gene were observed in the Simons Simplex Collection of unaffected siblings (de novo SNV $p$ value $<0.05$ ), and no rare type was reported in the Exome Variant Server. Recently 2 missense variants, p.Arg1170Cys and c.284C $>$ T (p.Ser95Leu), were found to have association with ASD patients [Homsy et al., 2015; Vissers et al., 2017].

The glycoprotein fibrillin-1 is an essential component of elastic fibers. In the brain, fibrillin-1 is an element of the capillary basement membrane, where it acts as an attachment protein between the 2 basal laminas [Tiedemann et al., 2005]. The blood-brain barrier ensures maintenance of the brain microenvironment and protects it from intrusion of toxic and inflammatory substances [Abbott et al., 2010]. Fibrillin-1 gene mutations give rise to misfolding and reduced secretion of the corresponding protein, resulting in a defective formation of the microfibrils [Schrijver et al., 1999; Whiteman et al., 2006], which in turn results in a basement membrane that is loosened and more permeable for macromolecules [Van der Donckt et al., 2015].

Fibrillin-1 also serves as a reservoir for the protein TGF- $\beta$, apart from providing structural support. Fibrillin-1 deficiency leads to an augmentation or dysregulation of TGF- $\beta$, which may trigger inflammation, accompanied by the release of proteases that degrade extracellular matrix components [Neptune et al., 2003; Judge et al., 2004; Matt et al., 2009; Holm et al., 2011]. In a study by Van der Donckt et al. [2015], fibrilin-1 gene-mutated mice models have elevated TGF- $\beta$ levels in the choroid plexus. As a consequence of this increase, expression of IL- $1 \beta$ and TNF- $\alpha$ as well as MMP- 2 and MMP-9 levels are enhanced providing further support to the hypothesis that TGF- $\beta$ acts as a mediator in inflammation [WyssCoray et al., 1997]. High TGF- $\beta$ levels have been linked to blood-brain barrier breakdown in inflammatory central nervous system diseases such as Alzheimer disease and multiple sclerosis [Lanz et al., 2010].
It has been shown that fibrillin controls the TGF- $\beta$ activity by acting as a structural platform for the latent TGF$\beta$-binding protein that sequesters and inactivates TGF- $\beta$, serving as a reserve pool for rapid injury response [Kaartinen and Warburton, 2003]. In addition, TGF- $\beta$ is involved in a link between the extracellular matrix and immune system disruption as it is a key immunomodulator, implicated not only within the joints in connective tissue disorders such as Ehlers-Danlos syndrome and MFS , but also in other organ systems such as lungs [Frischmeyer-Guerrerio et al., 2013]. Interestingly, several studies have found that TGF- $\beta 1$ levels were consistently lower in autism, which according to Ashwood et al. [2008] may help account for some of the immune dysregulation in the condition [Okada et al., 2007; Ashwood et al., 2008]. For these reasons, the TGF- $\beta$ pathway and upstream networks may be prime areas of study for future work into the overlapping etiologies of both connective tissue disorders and autism [Casanova et al., 2018].

\section{PLOD2}

PLOD2 (procollagen-lysine, 2-oxoglutarate 5-dioxygenase 2) encodes lysyl hydroxylase 2, which hydroxylates the lysine residues at the collagen type I carboxy-terminal telopeptide, allowing intermolecular cross-links between collagen fibrils [Uzawa et al., 1999]. This step is essential for an appropriate assembly of this protein, which is accumulated in several connective tissues, particularly in bone. Pathogenic variants in PLOD2 cause Bruck syndrome type 2(OMIM 609220), an autosomal recessive disease similar to osteogenesis imperfecta (OI) whose main characteristics are bone fractures and joint contractures that hinder mobility [Salo et al., 2008]. It may be due to this variant that the patients in our study manifested bone fragility. However, our patients do not present complete clinical features of Bruck syndrome. Studies have shown that protein encoded from the PLOD2 gene, interacts with COL1A1 and COL1A2 (https://string-db.org/cgi/network.pl?taskId=3Wk2wuvLqAYp) (Fig. 5). It is known that mutations in COL1A1 and COL1A2 lead to OI, a heterogeneous condition. Most of the OI-causing mutations (over $85 \%$ ) are in the type 1 collagen genes (COL1A1 or COL1A2) of which the replacement of a glycine amino acid in the $(\mathrm{Gly}-\mathrm{X}-\mathrm{Y})_{\mathrm{n}}$ repeating unit within the collagen triple helix are most common $(44,45)$. Using WES, Balasubramanian et al. [2018] identified variants of COL1A1 and COL1A2 in patients with autism and bone fragility. In 2 of the patients, pathogenic c. $902 \mathrm{G}>\mathrm{A}$ and c. $2533 \mathrm{G}>\mathrm{A}$ variants were identified separately in COL1A2. In the third patient, a de novo pathogenic variant in COL1A1,
68

Mol Syndromol 2020;11:62-72 DOI: $10.1159 / 000506530$
Farajzadeh Valilou et al. 


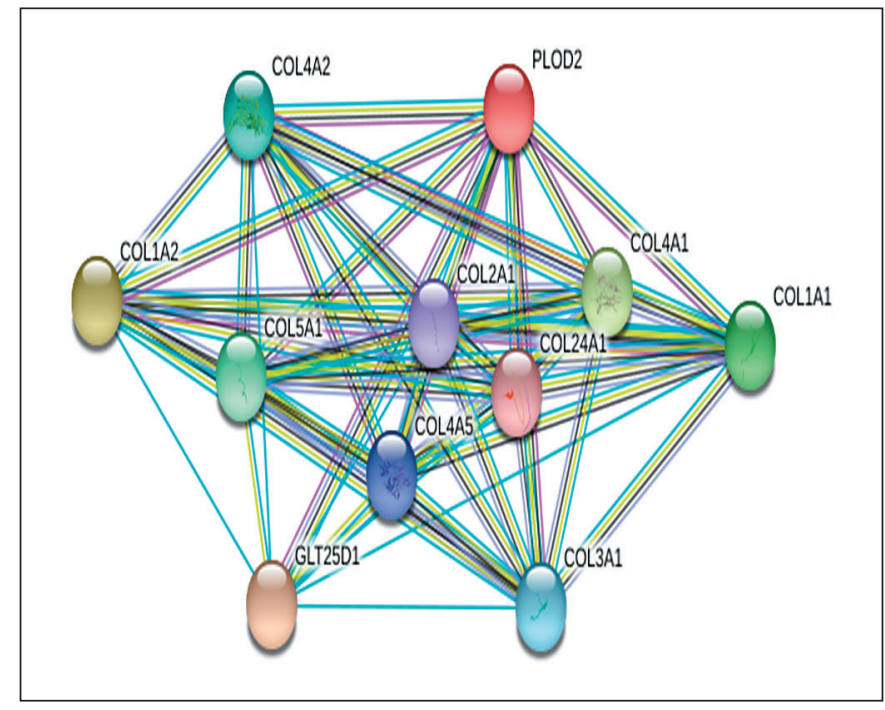

Fig. 5. PLOD2 interaction with various types of collagen proteins, especially COL1A1 and COL1A2.

c. $2282 \mathrm{G}>\mathrm{A}$, in exon $33 / 34$ was identified. Having found the identified COL1A1 and COL1A2 variants in patients with autism and bone fragility, and as far as PLOD2 interacts with COL1A1 and COL1A2, it can be inferred that pathogenic PLOD2 variants may have a deleterious effect on the normal function of collagen type 1 proteins. As a result, $P L O D 2$ may be involved in the development of not only bone fragility, but also autism. While the PLOD2 gene is described as a potential autism causative gene, further studies are necessary to strengthen our findings.

\section{TF}

The $T F$ gene encodes transferrin, a circulating serum protein responsible for delivering iron to cells (OMIM 190000) [Hershberger et al., 1991]. Each transferrin binds one ion of ferric iron at a time. Transferrin carries iron from the intestine, liver parenchymal cells, and the reticuloendothelial system to all proliferating cells in the body. This protein carries iron into cells via receptor-mediated endocytosis. Iron is dissociated from transferrin in a non-lysosomal acidic compartment of the cell. Supplying iron to cells is required for cell division. After dissociation of iron, transferrin and its receptor return to the extracellular environment and the intact cell membrane, respectively [Yang et al., 1984; Park et al., 1985; Bowman et al., 1988]. Until now, no variant in TF has been associated with autism, and the variant identified in this study (c.1027C > T; p.R343W) is the first variant in $T F$ which is reported in 2 patients with ASD.

Three Variants in a Family with ASD and Skeletal Problems
Transferrin has antioxidant activity in plasma, and its decreased levels have been associated with ASD patients with increased lipid peroxidation [Meguid et al., 2011]. Changes in plasma proteins due to electrophilic lipoxidation products potentially result in dysfunctional physiological consequences, including cell dysfunction, inflammatory response and apoptosis [Negre-Salvayre et al., 2008]. Furthermore, lipid peroxidation is a critical component of oxidative stress, a key process in the pathogenesis and progression of several human diseases, including neuropsychiatric disorders [De Felice et al., 2013; Csala et al., 2015; Halliwell and Gutteridge, 2015]. Evidence of serum transferrin oxidation could be described by a shortage in the defense mechanism against reactive oxygen species, as previously reported in autism [Meguid et al., 2011], knowing that it acts as an antioxidant by reducing the concentration of free ferrous iron. Cortelazzo et al. [2016] showed that the level of serum transferrin decreased in autistic individuals.

More importantly, it is well studied that iron plays a central role in many essential biological processes, including oxygen and electron transport [Aisen et al., 2001] and that it applies both positive and negative effects on brain function [Luck et al., 2013]. Therefore, iron deficiency (ID) and/or iron deficiency anemia (IDA) could possibly lead to the adverse effects on cognitive development and function observed in ASD children. With the task of binding iron (in the form of $\mathrm{Fe}^{3+}$ ) and safely delivering it to cells, the bilobal glycoprotein human serum transferrin comprises the most dynamic iron pool within the human body [Sheftel et al., 2012; Luck et al., 2013]. Following the production of transferrin in the liver and secretion into the blood, each lobe (referred to as the $\mathrm{N}$ and C-lobe) binds $\mathrm{Fe}^{3+}$ very tightly, yet reversibly [Aisen et al., 1978]. At the neutral pH of $\sim 7.4$ of the blood, ironbearing transferrin binds with nanomolar affinity to the transferrin receptor (TFR), located on the cell surface of all iron-requiring, i.e., activity dividing cells. The transferrin/TFR complex enters the cell by clathrin-dependent endocytosis [Luck et al., 2013]. It can be implied that a defect in TF may have an adverse effect on the normal function of the transferrin protein and may interfere with iron binding, leading to ID. Autism and ID could be associated with a common underlying genetic mechanism that has not yet been identified. On the other hand, iron is involved in brain monoamine systems including serotonin, dopamine, and noradrenaline, and the likely involvement of these systems in autism suggest that iron may influence monoamine-dependent neurotransmission [McCann and Ames, 2007; Herguner et al., 2012]. 
Although the genetic evidence supporting this has not yet been found, an association between ID and autism has been shown in several studies [Latif et al., 2002; Herguner et al., 2012; Sidrak et al., 2014; Gunes et al., 2017]. In South Wales, a routine blood investigation showed high prevalence of ID (52\%) [Latif et al., 2002]. Herguner et al. [2012] declared that in preschool children with autism ID was more common. In this study, $24.1 \%$ of the autistic children had ID and $15.5 \%$ had IDA with a ferritin cutoff of $<10 \mathrm{ng} / \mathrm{mL}$ for preschoolers and $<12 \mathrm{ng} / \mathrm{mL}$ for schoolage children. Also, anemia was defined as hemoglobin $<11.0 \mathrm{~g} / \mathrm{dL}$ for preschoolers and $<12.0 \mathrm{~g} / \mathrm{dL}$ for school-age children. Bilgic et al. [2010] demonstrated that one third of the Turkish preschool children with ASD had ID. It has been reported that the prevalence of ID in under 6-yearold autistic children was higher than autistic children over 6 years, and ID was found in 32\% of preschool autistic children. Similar to Herguner et al. [2012], 25\% of the children with ASD had ID, and 13\% had IDA in our study. Hemoglobin, hematocrit, and MCV levels of preschool patients with ASD were noticeably lower than school-age ASD patients [Bilgiç et al., 2010].

\section{Polygenic Mechanism in Autism}

Genetic studies and especially the development of high-throughput technologies have provided a large number of genes that constitute a comprehensive framework to better understand the complexity and heterogeneity in autism [de la Torre-Ubieta et al., 2016]. To date, hundreds of genes have been implicated in autism and their pathogenic roles in the development of autism are different. In addition to dominant, recessive, and gene-environment mechanisms, polygenic mechanisms in patients with autism have drawn attention to researchers in recent years [Chahrour et al., 2016]. In the study conducted by McKenna et al. [2018], polygenic risk for a complex developmental disorder was presented. They suggested that the probands' condition is due to the interaction of multiple rare variants in the context of a high-risk genetic background. This finding represents the multifactorial nature of genetic risk in neurodevelopmental disorders such as ASD [McKenna et al., 2018]. Multilocus genomic variation was also suggested in the study by Posey et al. [2017]. Using WES, they exhibited that structured clinical ontologies can be utilized to determine the degree of overlap between 2 mendelian diseases in the same patient. Similarly, in our study, contribution of variants in FBN1, PLOD2, and $T F$ suggests a polygenic mechanism. In this regard, confluence of these genes led to manifestation of a Marfan-like phenotype and skeletal problems in the patients.

\section{Conclusion and Outlook}

In our study, using WES and SNP array, we identified 3 pathogenic variants in $F B N 1, P L O D 2$, and $T F$ which are related to our patients' phenotypes. Regarding the skeletal features found in our patients, the role of the variants in FBN1 and PLOD2 would be of importance, most likely being causative of the phenotypes. Our results showed that despite direct or indirect association of these genes in neurodevelopmental disorders, no single variant adequately explains the probands' phenotypes. This finding represents the multifactorial nature of the genetic risk in ASD. Further research including animal model and functional studies of the 3 genes are necessary to reinforce and validate our findings and shed light on further understanding of the role and influence of these genes and also the polygenic mechanism in autism.

\section{Acknowledgments}

The authors thank the patients and their family for participating in this study. We are grateful to the Genetics Research Center and Sarem Hospital laboratory staff for assisting with the work. We sincerely thank Professor Andrew Crosby and Dr. Emma Baple from the Institute of Biomedical and Clinical Science, University of Exeter Medical School, and the RILD Wellcome Wolfson Centre, Exeter, UK for their scientific and laboratory support.

\section{Statement of Ethics}

The study was conducted at the Genetics Research Center at the University of Social Welfare and Rehabilitation Sciences and the Genetics Clinic of the Sarem Specialist Hospital from April 2016 to May 2018. Patient recruitment and all experimental protocols used in this study were in compliance with the Declaration of Helsinki and were approved by the Ethics Committee of the University of Social Welfare and Rehabilitation Sciences. Written informed consent was obtained from the parents prior to our investigations.

\section{Disclosure Statement}

The authors declare no conflicts of interest.

\section{Funding Sources}

We cordially thank the University of Social Welfare and Rehabilitation Sciences (grant No. 94/801/T/28882) and the Department of Human Genetics, University of Exeter for funding this project. 


\section{Author Contributions}

F.B., S.F.V.: study design; S.F.V.: manuscript preparation; A.A., F.B.: revision of the manuscript; S.F.V., A.A., S.G.F., A.N., J.K., M.F., F.B.: data analysis; S.F.V., M.P., J.K., M.F.: patient re- cruitment; S.F.V., M.P.: data collection; S.F.V., S.B.; laboratory work; Y.S., F.H., Z.H.: genetic counselling, and clinical examination; B.M.E., S.G.N.: ASD and psychological assessments; F.B.: grant application and receipt of the funding, supervision of the research.

\section{References}

-Abbott NJ, Patabendige AA, Dolman DE, Yusof SR, Begley DJ: Structure and function of the blood-brain barrier. Neurobiol Dis 37:13-25 (2010).

-Aisen P, Leibman A, Zweier J: Stoichiometric and site characteristics of the binding of iron to human transferrin. J Biol Chem 253:19301937 (1978).

-Aisen P, Enns C, Wessling-Resnick M: Chemistry and biology of eukaryotic iron metabolism. Int J Biochem Cell Biol 33:940-959 (2001).

American Psychiatric Association: Diagnostic and statistical manual of mental disorders (DSM-5), 5th ed (APA Publishing, Washington, DC 2013).

-Ashwood P, Enstrom A, Krakowiak P, Hertz-Picciotto I, Hansen RL, et al: Decreased transforming growth factor beta1 in autism: a potential link between immune dysregulation and impairment in clinical behavioral outcomes. J Neuroimmunol 204:149-153 (2008).

-Baio J, Wiggins L, Christensen DL, Maenner MJ, Daniels J, et al: Prevalence of autism spectrum disorder among children aged 8 years. MMWR Surveill Summ 67:1-23 (2018).

-Balasubramanian M, Jones R, Milne E, Marshall C, Arundel P, et al: Autism and heritable bone fragility: a true association? Bone Rep 8:156162 (2018).

- Bax DV, Bernard SE, Lomas A, Morgan A, Humphries J, et al: Cell adhesion to fibrillin-1 molecules and microfibrils is mediated by alpha 5 beta 1 and alpha v beta 3 integrins. J Biol Chem 278:34605-34616 (2003).

- Berg JM, Geschwind DH: Autism genetics: searching for specificity and convergence. Genome Biol 13:247 (2012).

-Bilgiç A, Gürkan K, Türkoğlu S, Akça ÖF, Kılıç BG, Uslu R: Iron deficiency in preschool children with autistic spectrum disorders. Res Autism Spect Dis 4:639-644 (2010).

Bowman BH, Yang FM, Adrian GS: Transferrin: evolution and genetic regulation of expression. Adv Genet 25:1-38 (1988).

-Casanova EL, Sharp JL, Edelson SM, Kelly DP, Casanova MF: A cohort study comparing women with autism spectrum disorder with and without generalized joint hypermobility. Behav Sci (Basel) 8:35 (2018).

-Chahrour M, O’Roak BJ, Santini E, Samaco RC, Kleiman RJ, Manzini MC: Current perspectives in autism spectrum disorder: from genes to therapy. J Neurosci 36:11402-11410 (2016).

-Cortelazzo A, De Felice C, Guerranti R, Signorini C, Leoncini S, et al: Expression and oxidative modifications of plasma proteins in autism spectrum disorders: interplay between inflammatory response and lipid peroxidation. Proteomics Clin Appl 10:1103-1112 (2016).

Csala M, Kardon T, Legeza B, Lizák B, Mandl J, et al: On the role of 4-hydroxynonenal in health and disease. Biochim Biophys Acta 1852:826838 (2015).

Dean JC: Marfan syndrome: clinical diagnosis and management. Eur J Hum Genet 15:724733 (2007).

De Felice C, Signorini C, Leoncini S, Pecorelli A, Durand T, et al: Fatty acids and autism spectrum disorders: the Rett syndrome conundrum. Food Nutri Sci 4:71-75 (2013).

de la Torre-Ubieta L, Won H, Stein JL, Geschwind $\mathrm{DH}$ : Advancing the understanding of autism disease mechanisms through genetics. Nat Med 22:345-361 (2016).

De Rubeis S, Buxbaum JD: Recent advances in the genetics of autism spectrum disorder. Curr Neurol Neurosci Rep 15:36 (2015).

-De Rubeis S, He X, Goldberg AP, Poultney CS, Samocha K, et al: Synaptic, transcriptional and chromatin genes disrupted in autism. Nature 515:209-215 (2014).

Devlin B, Scherer SW: Genetic architecture in autism spectrum disorder. Curr Opin Genet Dev 22:229-237 (2012).

Faivre L, Collod-Beroud G, Loeys B, Child A, Binquet $C$, et al: Effect of mutation type and location on clinical outcome in 1,013 probands with Marfan syndrome or related phenotypes and FBN1 mutations: an international study. Am J Hum Genet 81:454-466 (2007).

Frischmeyer-Guerrerio PA, Guerrerio AL, Oswald G, Chichester K, Myers L, et al: TGFbeta receptor mutations impose a strong predisposition for human allergic disease. Sci Transl Med 5:195ra194 (2013).

- Gunes S, Ekinci O, Celik T: Iron deficiency parameters in autism spectrum disorder: clinical correlates and associated factors. Ital J Pediatr 43:86 (2017).

Halliwell B, Gutteridge JM: Free Radicals in Biology and Medicine (Oxford University Press, New York 2015).

Herguner S, Kelesoglu FM, Tanidi, C, Copur M: Ferritin and iron levels in children with autistic disorder. Eur J Pediatr 171:143-146 (2012).

-Hershberger CL, Larson JL, Arnold B, Rosteck PR Jr, Williams P, et al: A cloned gene for human transferrin. Ann NY Acad Sci 646:140-154 (1991).

Holm TM, Habashi JP, Doyle JJ, Bedja D, Chen Y, et al: Noncanonical TGFbeta signaling con- tributes to aortic aneurysm progression in Marfan syndrome mice. Science 332:358-361 (2011).

Homsy J, Zaidi S, Shen Y, Ware JS, Samocha KE, et al: De novo mutations in congenital heart disease with neurodevelopmental and other congenital anomalies. Science 350:1262-1266 (2015).

-Iossifov I, Ronemus M, Levy D, Wang Z, Hakker $\mathrm{I}$, et al: De novo gene disruptions in children on the autistic spectrum. Neuron 74:285-299 (2012).

Iossifov I, O’Roak BJ, Sanders SJ, Ronemus M, Krumm N, et al: The contribution of de novo coding mutations to autism spectrum disorder. Nature 515:216-221 (2014).

-Jensen SA, Handford PA: New insights into the structure, assembly and biological roles of 10 $12 \mathrm{~nm}$ connective tissue microfibrils from fibrillin-1 studies. Biochem J 473:827-838 (2016).

Jovanovic J, Takagi J, Choulier L, Abrescia NG, Stuart DI, et al: alphaVbeta6 is a novel receptor for human fibrillin-1. Comparative studies of molecular determinants underlying integrin-rgd affinity and specificity. J Biol Chemistry 282:6743-6751 (2007).

Judge DP, Biery NJ, Keene DR, Geubtner J, Myers L, et al: Evidence for a critical contribution of haploinsufficiency in the complex pathogenesis of Marfan syndrome. J Clin Invest 114: 172-181 (2004).

Kaartinen V, Warburton D: Fibrillin controls TGFbeta activation. Nat Genet 33:331-332 (2003).

Krumm N, Turner TN, Baker C, Vives L, Mohajeri $\mathrm{K}$, et al: Excess of rare, inherited truncating mutations in autism. Nat Genet 47:582588 (2015).

Lanz TV, Ding Z, Ho PP, Luo J, Agrawal AN, et al: Angiotensin II sustains brain inflammation in mice via TGF- $\beta$. J Clin Invest 120: 2782-2794 (2010).

Latif A, Heinz P, Cook R: Iron deficiency in autism and Asperger syndrome. Autism 6:103114 (2002).

Luck AN, Bobst CE, Kaltashov IA, Mason AB: Human serum transferrin: is there a link among autism, high oxalate levels, and iron deficiency anemia? Biochemistry 52:8333-8341 (2013).

Lyall K, Croen L, Daniels J, Fallin MD, LaddAcosta C, et al: The changing epidemiology of autism spectrum disorders. Annu Rev Public Health 38:81-102 (2017).

Matt P, Schoenhoff F, Habashi J, Holm T, Van Erp C, et al: Circulating transforming growth factor-beta in Marfan syndrome. Circulation 120:526-532 (2009). 
McCann JC, Ames BN: An overview of evidence for a causal relation between iron deficiency during development and deficits in cognitive or behavioral function. Am J Clin Nutr 85: 931-945 (2007).

-McKenna B, Koomar T, Vervier K, Kremsreiter J, Michaelson JJ: Whole-genome sequencing in a family with twin boys with autism and intellectual disability suggests multimodal polygenic risk. Cold Spring Harb Mol Case Stud 4:a003285 (2018)

- Meguid NA, Dardir AA, Abdel-Raouf ER, Hashish A: Evaluation of oxidative stress in autism: defective antioxidant enzymes and increased lipid peroxidation. Biol Trace Elem Res 143: 58-65 (2011).

Negre-Salvayre A, Coatrieux C, Ingueneau C, Salvayre R: Advanced lipid peroxidation end products in oxidative damage to proteins. Potential role in diseases and therapeutic prospects for the inhibitors. Br J Pharmacol 153 . 6-20 (2008).

Neptune ER, Frischmeyer PA, Arking DE, Myers L, Bunton TE, et al: Dysregulation of TGF-beta activation contributes to pathogenesis in Marfan syndrome. Nat Genet 33:407-411 (2003).

> Okada K, Hashimoto K, Iwata Y, Nakamura K, Tsujii $M$, et al: Decreased serum levels of transforming growth factor-betal in patients with autism. Prog Neuropsychopharmacol Biol Psychiatry 31:187-190 (2007).

- Park I, Schaeffer E, Sidoli A, Baralle FE, Cohen GN, Zakin MM: Organization of the human transferrin gene: direct evidence that it originated by gene duplication. Proc Natl Acad Sci USA 82:3149-3153 (1985).
Posey JE, Harel T, Liu P, Rosenfeld JA, James RA, et al: Resolution of disease phenotypes resulting from multilocus genomic variation. $\mathrm{N}$ Engl J Med 376:21-31 (2017).

Reyes-Hernández OD, Palacios-Reyes C, ChávezOcaña S, Cortés-Malagón EM, AlonsoThemann PG, et al: Skeletal manifestations of Marfan syndrome associated to heterozygous R2726W FBN1 variant: sibling case report and literature review. BMC Musculoskelet Disord 17:79 (2016).

Salo AM, Cox H, Farndon P, Moss C, Grindulis $\mathrm{H}$, et al: A connective tissue disorder caused by mutations of the lysyl hydroxylase 3 gene. Am J Hum Genet 83:495-503 (2008).

$>$ Schrijver I, Liu W, Brenn T, Furthmayr H, Francke $\mathrm{U}$ : Cysteine substitutions in epidermal growth factor-like domains of fibrillin-1: distinct effects on biochemical and clinical phenotypes. Am J Hum Genet 65:1007-1020 (1999).

Sheftel AD, Mason AB, Ponka P: The long history of iron in the Universe and in health and disease. Biochim Biophys Acta 1820:161-187 (2012).

Sidrak S, Yoong T, Woolfenden S: Iron deficiency in children with global developmental delay and autism spectrum disorder. J Paediatr Child Health 50:356-361 (2014).

Tiedemann K, Sasaki T, Gustafsson E, Göhring W, Bätge B, et al: Microfibrils at basement membrane zones interact with perlecan via fibrillin-1. J Biol Chem 280:11404-11412 (2005).
Tucker T, Marra M, Friedman JM: Massively parallel sequencing: the next big thing in genetic medicine. Am J Hum Genet 85:142-154 (2009).

Uzawa K, Grzesik WJ, Nishiura T, Kuznetsov SA Robey PG, et al: Differential expression of human lysyl hydroxylase genes, lysine hydroxylation, and cross-linking of type I collagen during osteoblastic differentiation in vitro. J Bone Miner Res 14:1272-1280 (1999).

-Van der Donckt C, Roth L, Vanhoutte G, Blockx I, Bink DI, et al: Fibrillin-1 impairment enhances blood-brain barrier permeability and xanthoma formation in brains of apolipoprotein E-deficient mice. Neuroscience 295:1122 (2015).

Vissers L, van Nimwegen KJM, Schieving JH, Kamsteeg EJ, Kleefstra T, et al: A clinical utility study of exome sequencing versus conventional genetic testing in pediatric neurology. Genet Med 19:1055-1063 (2017).

Whiteman P, Hutchinson S, Handford PA: Fibril lin-1 misfolding and disease. Antioxid Redox Signal 8:338-346 (2006).

$\checkmark$ Wyss-Coray T, Borrow P, Brooker MJ, Mucke L: Astroglial overproduction of TGF- $\beta 1$ enhances inflammatory central nervous system disease in transgenic mice. J Neuroimmunol 77:45-50 (1997).

Yang F, Lum JB, McGill JR, Moore CM, Naylor SL, et al: Human transferrin: cDNA characterization and chromosomal localization. Proc Natl Acad Sci USA 81:2752-2756 (1984). 